\title{
Consistency of neutrino DIS and the present parton distribution functions.
}

\author{
Hannu Paukkunen* and Carlos A. Salgado \\ University of Santiago de Compostela \\ Departamento de Física de Partículas and IGFAE, Spain \\ Email: hannu.paukkunen@usc.es, carlos.salgado@usc.es
}

\begin{abstract}
We study the nuclear effects in the neutrino $\backslash$ anti-neutrino-nucleon deep inelastic scattering (DIS) by comparing the NuTeV, CDHSW, and CHORUS cross-sections from Iron and Lead targets to the predictions derived from the latest parton distribution functions (PDFs). The nuclear modifications found seem to display agreement with those in charged lepton DIS. Our study thus lends support to the consistency of employing neutrino data in global fits of PDFs.
\end{abstract}

35th International Conference of High Energy Physics

July 22-28, 2010

Paris, France

* Speaker. 


\section{Introduction}

Neutrino beam induced DIS provide information about the flavor decomposition of the PDFs that is difficult to obtain from the other available data. The weakness of the neutrino interactions, however, requires the usage of heavy targets - like huge blocks of Iron or Lead - in the experimental setup. It is well-known by now that the cross-sections involving bound nucleons are different from those involving free nucleons. Founded by the series of analyses in the past 12 years (see e.g. $[1,2,3]$ ), such differences have turned out to be factorizable - explainable by nuclear modifications solely in PDFs.

Interestingly, it was reported [4] that the $\mathrm{NuTeV} v \mathrm{Fe}$-data [5] implies that the nuclear effects in PDFs seem to be different in the neutrino-DIS than they are in the charged lepton DIS. Such a process-dependent difference would evidently ruin the factorization in the case of nuclear targets, and without factorization these data would be useless in improving the flavor decomposition of the free proton PDFs.

In this talk we summarize our analysis published in [6] which supports the factorization in neutrino-nucleon DIS and implies that the result of [4] is caused by looking solely the NuTeV data which seem to suffer from anomalous, neutrino-energy dependent normalization problems.

\section{Experimental Input}

The experimental data we use consists of neutrino $\backslash$ antineutrino DIS with nuclear targets from three independent experiments: NuTeV (Fe) [5], CDHSW (Fe) [7], and CHORUS (Pb) [8]. Importantly, we employ the published cross-sections instead of the structure functions extracted by the collaborations. These data sets provide enough kinematical overlap to also explore the mutual consistency of the different sets.

\section{Theoretical Framework}

Our analysis employs the $\overline{M S}$ variable flavor number scheme, its SACOT-prescription. The baseline free proton PDFs are taken from CTEQ6.6 [9], and the nuclear modifications from [1]. In addition, we account for the target mass (TM) correction, and correction for electroweak radiation (RAD) when calculating the cross-sections (see [6] for details). This is, in fact, the main reason why we prefer the cross-section data instead of the extracted structure functions: Both of these corrections clearly depend on the employed set of PDFs (see Table 1), and consequently the structure functions provided by the experiments depend on what they assumed about them.

\section{4. $\chi^{2}$-Values}

In Table 1 we provide the $\chi^{2} / N$-values measuring the agreement between the data and the theory. Evidently, in the case of CDHSW and CHORUS data, the full calculation with the CTEQ6.6 and the EPS09 gives excellent results with $\chi^{2} / N<0.8$. Also, the addition of the TM and RAD corrections improve the results. Only the $\mathrm{NuTeV}$ data behaves differently: Apart from good $\chi^{2} / N$, it appears strange why there is practically no effect whether we apply RAD and TM corrections or not. 


\begin{tabular}{ccc} 
Radiative and Target Mass corrections & CTEQ6.6 & CTEQ6.6×EPS09 \\
\hline \hline NuTeV & 1.51 & 1.05 \\
CHORUS & 1.15 & 0.79 \\
CDHSW & 1.10 & 0.71 \\
& & \\
No Radiative or Target Mass corrections & CTEQ6.6 & CTEQ6.6×EPS09 \\
\hline \hline NuTeV & 1.35 & 1.08 \\
CHORUS & 1.23 & 1.09 \\
CDHSW & 0.96 & 0.86
\end{tabular}

Table 1: The $\chi^{2} / N$-values computed using CTEQ6.6 with and without nuclear modification from EPS09. The numbers are given for calculations with and without the radiative and the target mass corrections.

\section{Shape Of The Nuclear Modifications}

Only from the bare $\chi^{2} / N$-values displayed in Table 1, a statistician would say that the calculations are in agreement with the data even without the nuclear corrections in PDFs. While probably true, the shape of the data - plotted as a function of Bjorken- $x$ - clearly shows the existence of the nuclear modifications. We plot two different ratios of cross-sections:

$$
R^{\text {CTEQ6.6 }} \equiv \frac{\sigma^{v, \bar{v}}(\text { Experimental })}{\sigma^{v, \bar{v}}(\text { CTEQ6.6 })}, \quad R^{\text {CTEQ6.6 } \times \text { EPS09 }} \equiv \frac{\sigma^{v, \bar{v}}(\text { CTEQ6.6 } \times \text { EPS09 })}{\sigma^{v, \bar{v}}(\text { CTEQ6.6 })} .
$$

In $R^{\text {CTEQ6.6 }}$ the denominator is stripped from the nuclear effects, and this ratio should therefore reflect the nuclear effects present in the experimental data. The other ratio $R^{\mathrm{CTEQ6} 6 \times \text { EPS09 }}$ is purely theoretical one and should agree with the former one if the nuclear effects in PDFs are universal.

In Figure 1, we plot the $Q^{2}$-averaged versions of these ratios as a function of $x$ for different neutrino energies $E_{\text {beam. }}$. We show the case of neutrino beam for all three data sets omitting the figures for antineutrinos as they are similar but with substantially larger uncertainties. The nuclear effects are clearly visible in the data: There is an excess around $x \cong 0.1$, the antishadowing, followed by a suppression at larger $x$, the EMC-effect. This shape is generally well reproduced by the nuclear effects from EPS09. However, in the $\mathrm{NuTeV}$ data there are evident, neutrino energy dependent fluctuations in the data. For example, the normalization of the data in panels with $E_{\text {beam }}=130 \mathrm{GeV}, 170 \mathrm{GeV}, 245 \mathrm{GeV}$ evidently deviate from the predictions, while e.g data in panels with $E_{\text {beam }}=85 \mathrm{GeV}, 95 \mathrm{GeV}, 190 \mathrm{GeV}$ are in a perfect agreement.

As the theoretical predictions depend only very weakly on the incident neutrino energy, these differences are impossible to fix only by changing the PDFs. That is, if the $\mathrm{NuTeV}$ data is used to extract the nuclear effects, the result will be some kind of compromise displaying tension between different subsets of data. This should be kept in mind when interpreting the results.

\section{Summary}

As a summary, we argue that the nuclear effects in PDFs extracted from other processes than neutrino DIS, agree also with the present neutrino data - only the $\mathrm{NuTeV}$ data, containing internal inconsistencies, display a tendency to different effects. Future neutrino data as e.g. those from the NOMAD collaboration may eventually help in settling down the issue. 

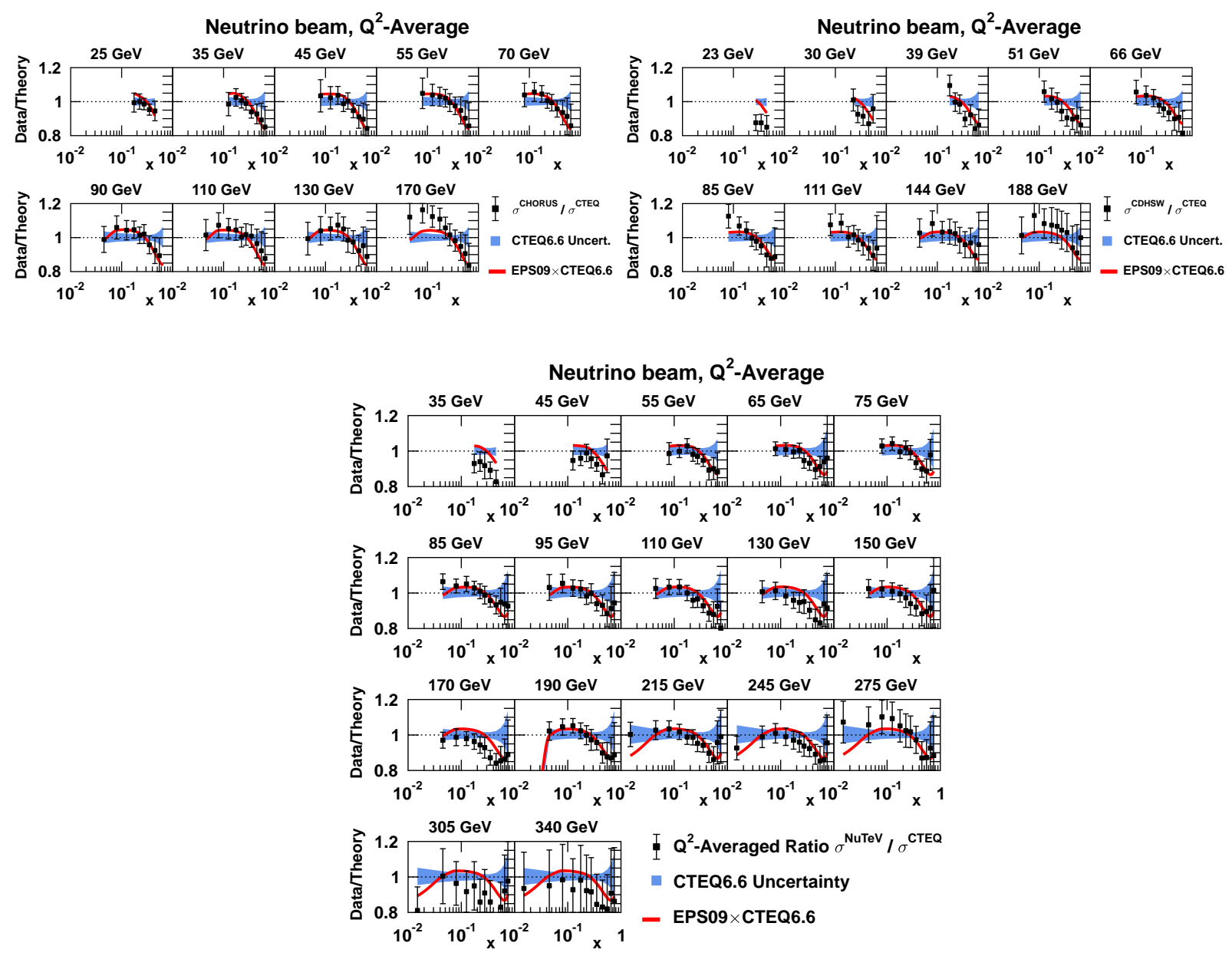

Figure 1: The $Q^{2}$-averaged CHORUS, CDHSW and NuTeV neutrino data.

\section{References}

[1] K. J. Eskola, H. Paukkunen and C. A. Salgado, JHEP 0904 (2009) 065 [arXiv:0902.4154 [hep-ph]].

[2] M. Hirai, S. Kumano and T. H. Nagai, Phys. Rev. C 76 (2007) 065207 [arXiv:0709.3038 [hep-ph]].

[3] D. de Florian and R. Sassot, Phys. Rev. D 69 (2004) 074028 [arXiv:hep-ph/0311227].

[4] I. Schienbein, J. Y. Yu, C. Keppel, J. G. Morfin, F. Olness and J. F. Owens, Phys. Rev. D 77 (2008) 054013 [arXiv:0710.4897 [hep-ph]].

[5] M. Tzanov et al. [NuTeV Collaboration], Phys. Rev. D 74 (2006) 012008 [arXiv:hep-ex/0509010].

[6] H. Paukkunen and C. A. Salgado, JHEP 1007 (2010) 032 [arXiv:1004.3140 [hep-ph]].

[7] J. P. Berge et al., Z. Phys. C 49 (1991) 187.

[8] G. Onengut et al. [CHORUS Collaboration], Phys. Lett. B 632 (2006) 65.

[9] P. M. Nadolsky et al., Phys. Rev. D 78 (2008) 013004 [arXiv:0802.0007 [hep-ph]]. 